\title{
EDART, a Discrete Algebraic Reconstructing Technique for Experimental Data Obtained with High Resolution Computed Tomography
}

\author{
L. Brabant* M. Dierick, E. Pauwels, M.N. Boone and L. Van Hoorebeke \\ Ghent University, Department of Physics and Astronomy \\ Proeftuinstraat 86, 9000 Gent, Belgium
}

September 23, 2013

\begin{abstract}
A novel reconstruction method to improve the image quality of three dimensional (3D) datasets, obtained with high resolution X-ray Computed Tomography $(\mu \mathrm{CT})$, for samples consisting of only one material and surrounding air is presented. It combines discrete tomography with iterative reconstruction algorithms, it is applicable for routine $\mu \mathrm{CT}$ applications and is referred to as the Experimental Discrete Algebraic Reconstruction Technique (EDART). A fast and intuitive method to estimate the attenuation coefficient and segmentation threshold, in case these are unknown, is included. Experimental results illustrate that EDART allows improving the reconstruction quality relatively to standard iterative reconstruction when few projections are available, without significantly increasing the reconstruction time.
\end{abstract}

keywords: discrete tomography, high resolution CT, iterative reconstruction

\section{Introduction}

High resolution X-ray computed tomography $(\mu \mathrm{CT})$ is a powerful, non-destructive technique which allows for the investigation of the internal structure of objects.

An important challenge lies in the correct reconstruction of the projection data obtained with $(\mu \mathrm{CT})$. As the datasets obtained with laboratory based $\mu \mathrm{CT}$ are of substantial size, it is a challenging problem to obtain accurate reconstructions in a reasonable amount of time.

To obtain the optimal reconstruction quality, all the information that is available

*Corresponding author. E-mail Loes.Brabant@ugent.be Tel. +32 92646611 Fax +32 9264 6697 
about the sample should be used during the reconstruction. Often tomography is applied on sample consisting of only one or a limited number of materials, which is referred to as discrete tomography [1. In discrete tomography the purpose is to obtain more accurate reconstructions by using the prior knowledge such as the number of materials and the attenuation coefficients of these materials. It is often used to reduce the number of necessary projections. There exist several algorithms for discrete tomography, e.g. 2, 3, 4, 5, 6, 7, 8, 9, For medical applications, most algorithms assume that the value of the attenuation coefficient is known in advance, because the scanned materials are known in advance. In laboratory based tomography, all kinds of samples are scanned and the attenuation coefficient(s) of the material(s) often are unknown. Additionally, most algorithms for discrete tomography are computationally demanding and time consuming, particularly for 3D volumes. A typical dataset obtained with $\mu \mathrm{CT}$ is of substantial size, so a fast computationally efficient algorithm is required in practical applications.

Recently, Batenburg and Sijbers proposed a new reconstruction algorithm for discrete tomography, called DART (Discrete Algebraic Reconstruction Technique; 10]) which had several practical applications [11, 12, 13. DART combines discrete reconstruction with iterative reconstruction algorithms. Iterative reconstruction algorithms [14, 15, 16, 17, 18, 19, 20, 21, 22, 23, 24, 25] are becoming a more widely used alternative for analytical reconstruction algorithms, such as the algorithm of Feldkamp, David and Kress (FDK) 26, which are based on filtered back projections. These algorithms can be used to reduce artifacts such as cone beam artifacts 27, 28, metal artifacts [29, 30, and beam hardening artifacts [31, 32, 33. Moreover, they can provide better noise handling and reconstructions of higher quality when only a limited number of projections are available 34, 35, 36, 37. With DART it is possible to obtain more accurate reconstructions for samples consisting of a number of discrete materials, even when the number of projections is limited.

Although DART can produce reconstructions of much better quality in comparison with regular iterative reconstruction algorithms, the DART algorithm requires accurate knowledge about the attenuation coefficient(s) and the threshold(s) in order to produce accurate results. Several methods for the determination of the attenuation coefficient and threshold are available [38, 39, 40. However, most algorithms are very time consuming or are not applicable for every type of sample, which makes them not always practically feasible for routine $\mu \mathrm{CT}$ applications. Even when the attenuation coefficient is known, the reconstruction times of DART can increase because several discretization and reconstruction steps are needed. The reconstruction times reported in 40 indicate that the computational time for DART is significantly larger in comparison with the reconstruction time of classical iterative reconstruction, especially for datasets where the attenuation coefficient has to be estimated.

The question arises whether it would be possible to obtain a better result in comparison with classical iterative reconstruction algorithms while keeping the reconstruction time in the same order of magnitude. In this paper an algorithm inspired by the DART algorithm will be presented: the Experimental Discrete 
Algebraic Reconstruction Technique (EDART). With this algorithm it is not our goal to obtain the best possible result, but to obtain a better result in comparison with standard iterative reconstruction techniques without significantly increasing the reconstruction time. The EDART algorithm will be explained for reconstruction of 3D datasets consisting of only one material and surrounding air, but it can be extended towards datasets consisting out of more materials. Additionally, a fast and intuitive way to estimate the attenuation coefficient and threshold in the dataset consisting of one material and surrounding air is presented.

\section{Materials and methods}

In this section, first the basic concepts of iterative reconstruction are reviewed. The EDART algorithm will be presented, as well as a fast and intuitive way to determine the attenuation coefficient of the material of the sample in case this is not known in advance. Subsequently, the samples and parameters and set-up used for scanning them are described and finally the methods for evaluations of the results are discussed.

\subsection{Iterative Reconstruction}

Iterative reconstruction algorithms consider the reconstruction process as the optimization of a discrete representation of the object $f(x, y, z)$ in order to satisfy a system of equations that describes the imaging modality. The object function is represented on a $3 \mathrm{D}$ grid of $N$ voxels (3D equivalent of pixels) corresponding to function values $f_{j}$ with $j=1,2, \ldots, N$. The line integral corresponding to a detector pixel can be written in a discrete form, $p_{i}$, which is called a ray sum. Algebraic reconstruction algorithms try to find a solution to the linear equation system:

$$
\mathbf{W} \cdot \mathbf{f}=\mathbf{p} \text {. }
$$

f is the $(N \times 1)$ column vector representing the function values $f_{j}$ and $\mathbf{p}$ is a $(M \times 1)$ column vector containing the ray sums $p_{i}$, with $M=R M_{p}$ the total number of measured ray sums in all the projection images; $R$ represents the number of projections and $M_{p}$ the number of detector pixels. $\mathbf{W}$ is the $(M \times N)$ matrix of the weights $w_{i j}$, which represent the contribution of the $j$-th voxel to the $i$-th ray sum.. In this paper the SART algorithm [15] is used, but both DART and EDART can be used with different algebraic reconstruction techniques. With SART the update process is described by [15]:

$$
f_{j}^{(k+1)}=f_{j}^{k}+\lambda \frac{\sum_{p_{i} \in P_{\phi}}\left(\frac{p_{i}-\sum_{n=0}^{N} w_{i n} f_{n}^{k}}{\sum_{n=0}^{N} w_{i n}}\right) w_{i j}}{\sum_{p_{i} \in P_{\phi}} w_{i j}} .
$$

Where $f_{j}^{k}$ represents the linear attenuation coefficient of voxel $j$ after the $k$-th iteration, $\lambda$ is a relaxation parameter and $P_{\phi}$ is the projection with projection angle $\phi$. One SART iteration uses every projection once. 


\subsection{EDART}

The Experimental Discrete Algebraic Reconstruction Technique (EDART) was developed because we needed an algorithm which improves the reconstruction quality by taking the discrete nature of the sample into account, without significantly increasing reconstruction time. With EDART we will try to improve the reconstruction quality by using only one discretization step. These are the different steps in the EDART algorithm in case the attenuation coefficient $\mu$ of the material of the sample and a realistic threshold $T$ are known in advance, in comparison with a SART reconstruction with $2 \times N_{i t}$ iterations:

1. Perform a SART reconstruction with $N_{i t}$ iterations.

2. Apply a smoothing algorithm to reduce the noise (we use a median filter, but other types of filter can be used as well).

3. Segment the volume. Every voxel with a value lower than $T$ is set to zero and the others are set to $\mu$.

4. The segmented volume is used as input for a new SART reconstruction with $N_{i t}$ iterations.

5. The result is smoothed and segmented.

In this way the total number of iterations remains the same as in the SART algorithm. Note that each SART iteration uses every projection once. The only overhead which is introduced by using EDART is applying the smoothing and the discretization step.

The necessary number of iterations $N_{i t}$ will of course depend on the available number of projections. When only a limited number of projections are available the reconstruction quality can be improved by increasing the number of iterations. However, increasing $N_{i t}$ will also increase the reconstruction time, so the number of iterations should only be increased when it has an effect on the reconstruction quality. To determine if the reconstruction is affected by changing the number of iterations we evaluated whether the histogram changed significantly for a single slice reconstruction when the number of iterations was changed. Several single slice reconstructions with increasing number of iterations were performed and Otsu's threshold [41] was determined for each reconstruction. If the determined threshold did not vary significantly when the number of iterations was increased, the current number of iterations was set as $N_{i t}$.

\subsection{Determining the attenuation coefficient and threshold}

In the previous section we assumed that the attenuation coefficient and threshold of the sample are known in advance. In case of non-medical experimental datasets this is often not the case. Moreover, when using the experimental data artifacts can occur, the projection images contain noise and the samples are often not completely discrete. As a result, there will be a variation in the reconstructed attenuation coefficients of voxels belonging to the same material. 
In this case a virtual attenuation coefficient $\mu$ for the material which results in the best reconstruction quality needs to be determined. In this section a method to estimate $\mu$ and $T$ is presented. When the EDART algorithm is used in combination with this method we will refer to this as EDART(calc), while the EDART algorithm in combination with known $\mu$ and $T$ is referred to as $\operatorname{EDART}($ known).

In the case of a discrete sample which consists only of one material, a single slice of the sample can be used to estimate the attenuation coefficient and threshold. We select an initial value $\mu^{a}$ and perform the steps listed below to determine a new value for the attenuation coefficient, $\mu^{b}$. To determine the thresholds $T^{a}$ and $T^{b}$ we used Otsu's method [41] but other algorithms can be used as well.

a) Perform a SART reconstruction with $N_{i t}$ iterations and apply a smoothing operation.

b) Determine a threshold $T^{a}$ to segment the volume; every voxel with a value lower than $T^{a}$ is set to zero and the others are set to $\mu^{a}$

c) Use the segmented slice as input for a new SART reconstruction with $N_{i t}$ iterations and apply a smoothing operation.

d) Determine a new threshold for the sample, $T^{b}$.

e) Determine a new value for the attenuation coefficient of the sample, $\mu^{b}$. This is done by selecting the histogram bin with the highest peak value in the range $\left[T, h_{\max }\right]$, with $h_{\max }$ the highest value in the histogram range.

During an iterative reconstruction, the difference between the measured and the calculated ray sum is first multiplied with a relaxation parameter and then backprojected. This difference should be minimal. If the initial solution is not good, the difference will be rather large and the initial solution will be corrected during this backprojection. As a result, if the initial value $\mu^{a}$ is too small, the new attenuation coefficient $\mu^{b}$ will be larger than $\mu^{a}$. If we start with a $\mu^{a}$ which is too large, the resulting $\mu^{b}$ will be smaller. If the value for $\mu^{a}$ is close to the real value for $\mu$ the difference between $\mu^{a}$ and $\mu^{b}$ will be minimal. Therefore we propose a new algorithm for determining $\mu$ and $T$, of which the pseudo code is listed below.

This algorithm starts with an initial guess value which is set as central value $\mu_{\mathrm{c}} . \quad n$ is an integer value (typically $n \in[2,4]$ ) which is chosen to allow a variation around $\mu_{\mathrm{c}}$ and $\mu_{\text {step }}$ is a relatively large step value (typically such that $\left.\frac{\mu_{c}}{\mu_{\text {step }}} \in[5,10]\right)$ for stepwise changing $\mu_{i}$, with $i$ the index of the calculation. The value of $\mu_{\mathrm{c}}$ is dependent of the sample, if the initial value of $\mu_{c}$ turns out to be too small or too large it is changed in line 16 or line 29 of the algorithm respectively. $\epsilon_{\text {stop }}$ is a chosen stop value (typically $\epsilon_{\text {stop }} \in[0.001,0.020]$ ); a smaller value of $\epsilon_{\text {stop }}$ will increase the accuracy of the resulting $\mu$ and $T$. Lines 4 until 7 ensure that $\mu_{0}^{a}$ is positive. In lines 8 until $112 n+1$ values of $\mu^{a}$ and $\mu^{b}$ are determined. If the conditions in line 14 and 20 are fulfilled the stop criterion 


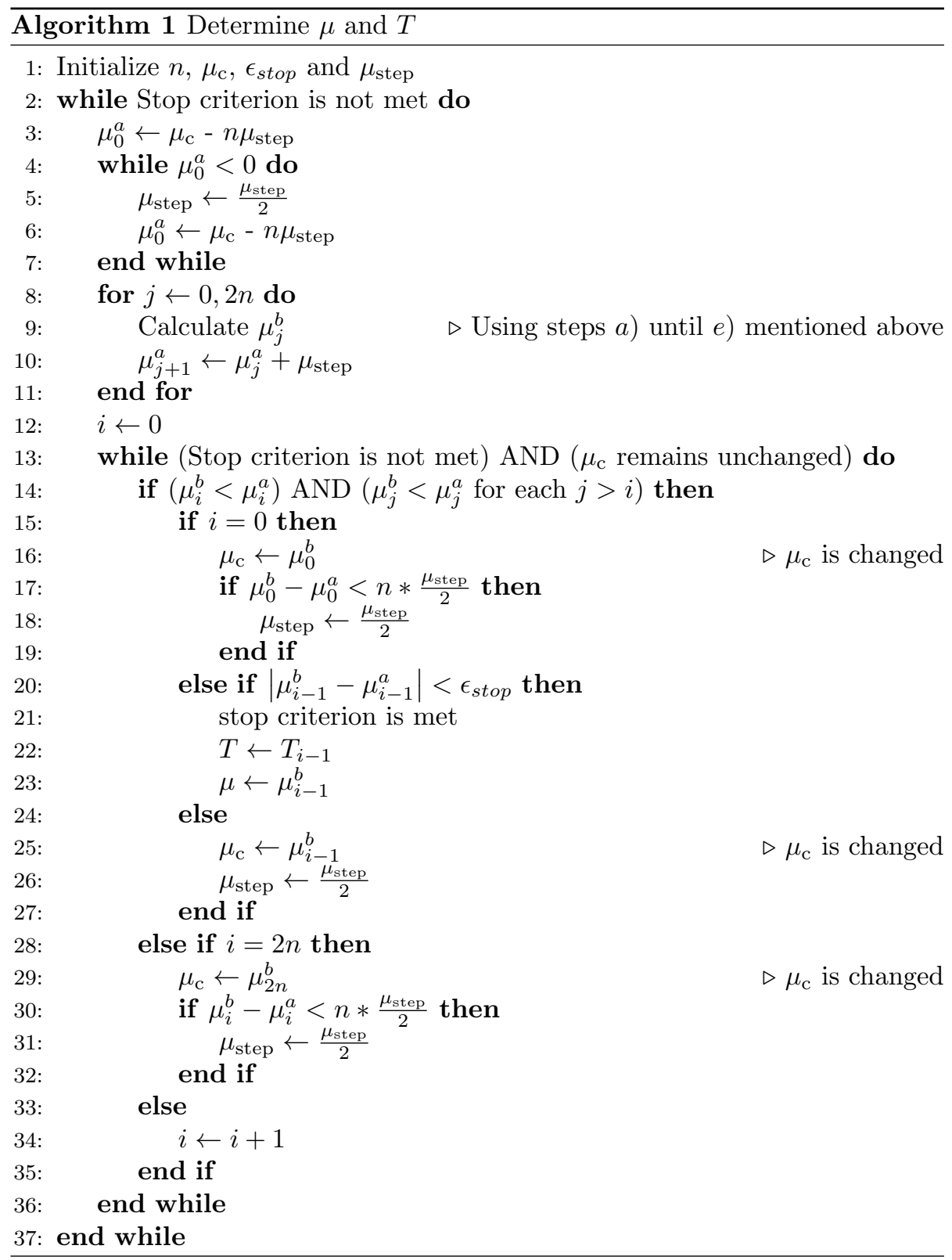


is met and the values of $\mu$ and $T$ are returned. Every time a new reconstruction is executed the result can be visualized so the user can also visually evaluate the results.

When using this approach, two parameters $\mu$ and $T$ are determined based on the evolution of one parameter. This is why we used the evolution around a central value $\mu_{c}$. Indeed, it can happen that $\mu_{i}^{a}$ generates a lower $\mu_{i}^{b}$ only because the threshold is changed to compensate for the difference between the measured and the calculated ray sum and not because the optimal $\mu$ is smaller than $\mu^{a}$. In this case a local decrease will occur and in one of the next steps $(j=i+1, i+2, .$. there will be an increase: $\mu_{j}^{b}>\mu_{j}^{a}$. Thus it is very important to ensure that the selected $i$ for which a decrease occurs indicates a global decrease, this means that for each $j>i: \mu_{j}^{b}<\mu_{j}^{a}$, which is the second condition in line 14 .

The EDART method was presented for the reconstruction of samples consisting out of one material and surrounding air, however it can be extended towards multi-material samples. If the attenuation coefficient and threshold are known in advance, the extension is trivial, if this is not the case the method for calculating $\mu$ and $T$ needs to be extended as well. For the segmentation a multi-level thresholding technique should be used, for example the one mentioned in [42]. Then each $\mu(i)$ can be calculated by applying the method mentioned above in the interval $[T(i-1), T(i)]$.

\subsection{Samples}

EDART and SART reconstructions of three samples with different structures are compared: a toy sample, an aluminum foam sample and a sample of four cookies. Micro-CT scanning was performed using the micro-CT scanner setup at UGCT [43. This flexible setup allows selecting the optimal scanning parameters (X-ray source, detector, filters...) to maximize resolution and image quality depending on the sample. For all the samples the Feinfocus FXE 160 X-ray tube was used. For the toy and the aluminum foam sample this was combined with a Varian PaxScan 2520V flat panel detector and for the sample of four cookies with a PerkinElmer 1620 CN3 CS flat panel detector. A $3 \mathrm{~mm}$ aluminum filter was used for the aluminum foam sample and a $1 \mathrm{~mm}$ aluminum filter for the other samples. The X-ray tube was operated at $80 \mathrm{kV}$ for the sample of cookies, $100 \mathrm{kV}$ for the aluminum foam sample and at $120 \mathrm{kV}$ for the toy sample. The projection images were cropped and rebinned, resulting in 1000 projections of $910 \times 725$ pixels for the toy sample, 1500 projections of 940 x 748 pixels for the aluminum foam sample and 1200 projections of 1019 x 519 pixels for the sample of cookies. The resulting voxel pitch was $34 \mu \mathrm{m}$ for the toy sample, $67 \mu \mathrm{m}$ for the aluminum foam sample and $56 \mu \mathrm{m}$ for the sample of cookies.

A virtual phantom of the toy sample was created as well: the toy sample was reconstructed with the SART algorithm and binarized using a global thresholding algorithm. A virtual attenuation coefficient of $1.0 \mathrm{~cm}^{-1}$ was assigned to the material and the 512 projections were simulated with the in house developed Projection Simulator [28. The advantage of using a simulation is that the 
exact solution, the ground truth, is known in advance, which makes it easier to compare different algorithms. Simulations do not suffer from artifacts (for example arising from the polychromaticity of the beam which is not taken into account during the reconstruction). Another advantage is that the attenuation coefficient is known, which also allows testing the above mentioned algorithm for determining the attenuation coefficient.

\subsection{Evaluation}

The results can be visually evaluated, or in case a ground truth is available, the accuracy of the reconstructions can be quantified using the relative Number of Misclassified voxels (rNMV), which is defined as:

$$
r N M V=\frac{M V}{V o I},
$$

with $M V$ the total number of Misclassified Voxels, i.e. the total number of voxels that are classified in as being part of a different phase than in ground truth volume and $V o I$ the Voxels of Interest, i.e. the total number of voxels belonging to the phase of interest (either the material of the sample or the air) in the ground truth volume.

\section{Results and Discussion}

In this section the reconstruction results using EDART and SART with different number of projections will be described for three samples: the toy sample, the aluminum foam sample and the cookie sample. At first the reconstruction of the virtual toy phantom will be discussed. In this case the exact solution is known. For the experimental datasets the exact solution is not known, in these cases a SART reconstruction using a large number of projections is binarized with a global thresholding method using Otsu's threshold. This binary solution is considered to be the ground truth.

\subsection{Virtual Toy phantom}

For the virtual toy phantom we are most interested in the cavity inside the sample, as this is invisible for the outside observer. In Figure 1 the cavity in the phantom is visualized with VGStudio Max 2.0. The sample was reconstructed with SART and EDART(known) using 8, 16, 32 and 64 projections with $\mu=1.0 \mathrm{~cm}^{-1}$ and $T=0.5 \mathrm{~cm}^{-1}$. In each case the projections were evenly distributed over $360^{\circ}$. Additionally the EDART(calc) algorithm was used to estimate $\mu$ and $T$. In Table 1 the resulting values for the estimated $\mu$ and $T$ are shown for the reconstructions using different number of projections $N_{p}$. For 8 and 16 projections $N_{i t}=20$ was used and for $N_{p}=32$ and $N_{p}=64, N_{i t}=10$ was used for the EDART reconstructions. To compare reconstructions with the same amount of iterations, $2 \times N_{i t}$ iterations were used for the SART reconstructions. The SART reconstructions were segmented by using Otsu's threshold, in 


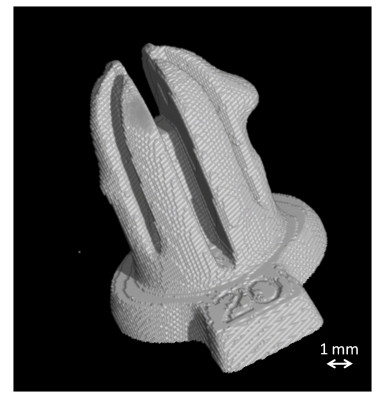

Figure 1: Cavity in the virtual toy phantom. The scale bar indicates the scale of the real sample of which this phantom was created.

Table 1: Calculated $\mu$ and $T$ for the virtual toy phantom in the case of different number of projections. The scale bar indicates the scale of the real sample of which this phantom was created.

\begin{tabular}{ccc}
\hline$N_{p}$ & $\mu\left(\mathrm{cm}^{-1}\right)$ & $T\left(\mathrm{~cm}^{-1}\right)$ \\
\hline 8 & 0.904 & 0.434 \\
16 & 0.991 & 0.475 \\
32 & 1.007 & 0.480 \\
64 & 1.000 & 0.493 \\
\hline
\end{tabular}

order to be able to compare the discrete images. In Figure 2 the $r N M V$ for the different reconstructions of the cavity is shown; to determine the cavity region, the same region as in Figure 1 was extracted from the different reconstructions and the voxels belonging to the air phase were visualized. Figure 3 shows the EDART(calc) and SART reconstructions of the cavity using 8, 16 and 32 projections, visualized with VGStudio Max 2.0. From Figure 2 it can be observed that the $r N M V$ is higher for the SART than for the EDART reconstruction. Especially for the lower number of projections it is clear that EDART performs better than SART, when more projections are available the difference decreases. The $r N M V$ of the EDART(calc) reconstructions is slightly higher than that of the EDART(known) reconstructions, but still lower than the SART reconstructions. From Figure 3 it is clear that a larger part of the cavity can be retrieved with the EDART algorithm than with the SART algorithm. Especially more of the pore voxels at the top of the sample can be identified with EDART. Note that these pore voxels are the most difficult to identify because they are fewer in number and surrounded by more material-voxels than the pore voxels at the bottom of the sample. Additionally, more details can be distinguished with the EDART algorithm: the inscription of the number 20 at the bottom of the sample can already be distinguished with the EDART algorithm when only 8 projections are available; for the SART algorithm 32 projections are necessary, and even then the number is better defined in the EDART reconstruction. 


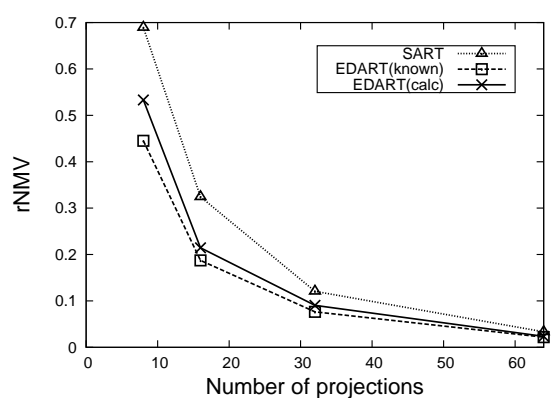

Figure 2: The $r N M V$ of the cavity in the toy phantom for different reconstruction techniques with various number of projections: SART, EDART with known $\mu$ and $T$ (EDART(known)), and EDART with the calculated values for $\mu$ and $T$ (EDART(calc)), as mentioned in Table 1 .
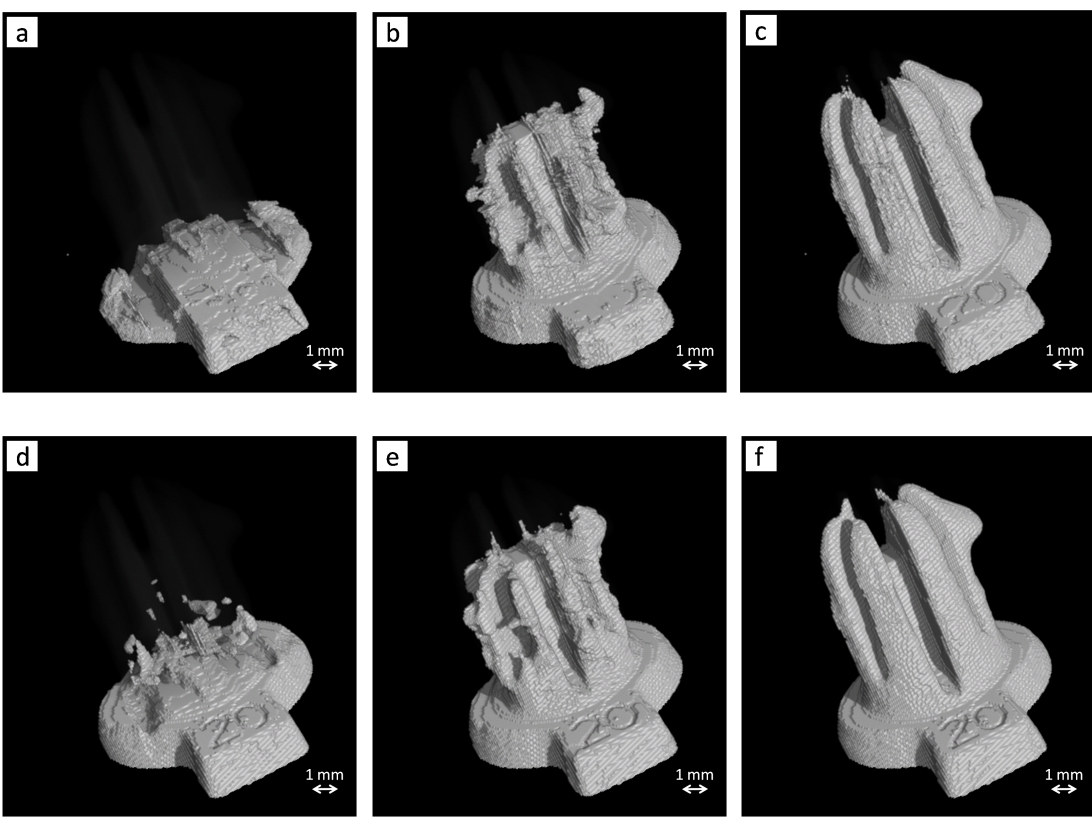

Figure 3: Reconstructed cavity (same region as in Figure 1) in the virtual toy sample in case of SART with $N_{p}=8$ (a), $N_{p}=16$ (b) and $N_{p}=32$ (c), and EDART(calc) with $N_{p}=8(\mathrm{~d}), N_{p}=16$ (e) and $N_{p}=32$ (f). The scale bar indicates the scale of the real sample of which this phantom was created. 

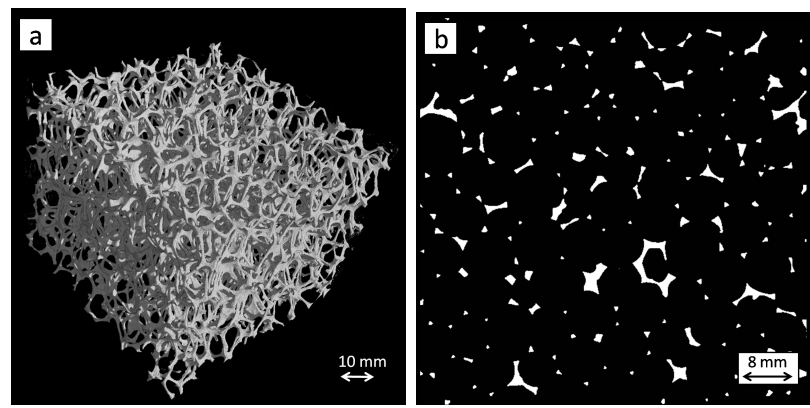

Figure 4: 3D rendering visualized with VGStudio Max 2.0 (a) and a reconstructed slice (b) of the aluminum foam sample

\subsection{Experimental samples}

The EDART algorithm was tested on three experimental datasets with different structures. Each sample consists of one material and surrounding air: an aluminum foam sample, a sample of four cookies and a toy sample (of which a phantom was created in the previous section). As a ground truth for these samples a SART reconstruction with 2 iterations using 1000 projections in case of the toy sample, 1500 projections for the aluminum foam sample and 1200 projections for the sample of the four cookies was used. This reconstruction was first smoothed and then binarized with a global threshold algorithm using Otsu's threshold. In Figure 4(a) and 4(b) a 3D rendering visualized with VGStudio Max 2.0 and a reconstructed slice of the binarized aluminum foam are shown, respectively. Figure 5(a) and 5(b) show a 3D rendering visualized with VGStudio Max 2.0 and a reconstructed slice of the sample of cookies, respectively; the rectangle indicates a porous region in one of the cookies. For both of these samples the phase of interest is the material phase so this is visualized. For the toy sample we are again most interested in the cavity, the 3D rendering of this cavity is not shown as it is similar to the one shown in Figure 1

For these samples, $\mu$ and $T$ are not known, so the EDART(calc) algorithm is used for the reconstructions. The samples were reconstructed using different number of projections $N_{p}$. In Table 2 the number of iterations $N_{i t}$ and the resulting $\mu$ and $T$ are shown for the different samples reconstructed with different $N_{p}$. In order to compare reconstructions with the same number of iterations $2 \times N_{i t}$ iterations were used for the SART reconstructions. The SART reconstructions were binarized with Otsu's threshold as global threshold equal to $0.327 \mathrm{~cm}^{-1}, 0.134$ $\mathrm{cm}^{-1}$ and $0.097 \mathrm{~cm}^{-1}$ for the foam, cookie and toy sample, respectively. The SART reconstruction time for using all available projections was approximately 30 minutes for the sample of cookies and the toy sample and 45 minutes for the aluminum foam sample (exact reconstruction times are not given because they depend on the implementation of the code and the used computational hardware). Reducing the number of projections reduces these reconstruction 

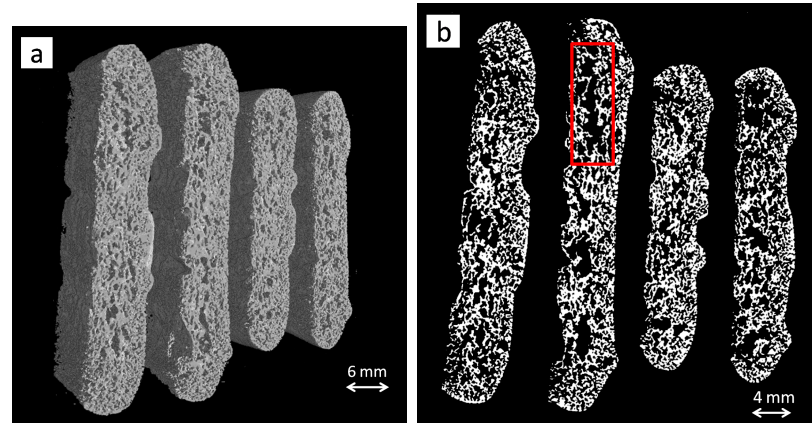

Figure 5: 3D rendering visualized with VGStudio Max 2.0 (a) and a reconstructed slice (b) of the sample of four cookies. The rectangle indicates a porous region in one of the cookies

times as well. The EDART reconstructions resulted in a constant increase of reconstruction time (independent of the number of projections), which varied between 5 and 7 minutes, in comparison with the SART reconstructions, including the time to calculate $\mu$ and $T$. In Figure 6(a) 6(b) and 6(c) the $r N M V$ for the SART and $\operatorname{EDAR}$ (calc) reconstructions is shown for the aluminum foam sample, the sample of cookies and the cavity in the toy sample, respectively.

A trend can be observed in Table 2, as the number of projections decreases, the calculated values for $\mu$ and $T$ decrease as well. This trend is most significant for the sample of cookies. The cookies have a very fine structure with many boundaries, and as the number of projection decreases, more information which is necessary for an accurate reconstruction is missing. As a result, the boundaries of the reconstructed sample will be less well-defined and the boundaries will be smeared out over multiple voxels because of the partial volume effect, which will cause the attenuation coefficient to vary for different voxels. The attenuation coefficient is no longer discrete and the calculated attenuation coefficient will be smaller because of the partial volume effect. Because this sample has many boundaries, this effect is significant.

From Figure 6 it can be observed that the $r N M V$ of the EDART(calc) reconstructions is lower than that of the SART reconstructions in case there are few projections available. If the number of projections increases, the difference in $r N M V$ decreases. For the sample of cookies the SART reconstruction using 600 projections has even a slightly lower rNMV than the EDART(calc) reconstructions. The better results for the EDART(calc) reconstructions in case the number of available projections is limited can also be visually observed. Figure 7 shows the same reconstructed cross section of the aluminum foam sample as that in Figure 4(b) in case of SART using $N_{p}=50$ (a), $N_{p}=150$ (b) and EDART(calc) using $N_{p}=50$ (c) and $N_{p}=150$ (d). It can be observed that the material phase is significantly overestimated in case of the SART reconstructions. Figure 8 shows the same reconstructed cross section of the sample of 
Table 2: $N_{i t}$, calculated $\mu$ and calculated $T$ for the aluminum foam sample, the sample of four cookies and the toy sample in the case of different number of projections $N_{p}$

\begin{tabular}{ccccc}
\hline Sample & $N_{p}$ & $N_{i t}$ & $\mu\left(\mathrm{cm}^{-1}\right)$ & $T\left(\mathrm{~cm}^{-1}\right)$ \\
\hline Aluminum foam & 20 & 10 & 0.527 & 0.257 \\
& 50 & 5 & 0.623 & 0.310 \\
& 100 & 5 & 0.637 & 0.336 \\
& 150 & 5 & 0.647 & 0.349 \\
& 250 & 5 & 0.636 & 0.341 \\
& 500 & 3 & 0.637 & 0.329 \\
Cookies & 10 & 10 & 0.151 & 0.098 \\
& 20 & 10 & 0.187 & 0.100 \\
& 50 & 5 & 0.189 & 0.098 \\
Toy & 100 & 5 & 0.211 & 0.114 \\
& 200 & 5 & 0.232 & 0.121 \\
& 400 & 3 & 0.241 & 0.133 \\
& 600 & 2 & 0.243 & 0.134 \\
& 5 & 10 & 0.186 & 0.083 \\
& 10 & 5 & 0.189 & 0.089 \\
& 20 & 5 & 0.196 & 0.093 \\
& 50 & 5 & 0.198 & 0.099 \\
& 100 & 5 & 0.199 & 0.099 \\
\hline
\end{tabular}

cookies as that in Figure 5(b) in case of SART using $N_{p}=20(\mathrm{a}), N_{p}=50$ (b) and EDART(calc) using $N_{p}=20$ (c) and $N_{p}=50$ (d). Again the material phase is overestimated in case of the SART reconstruction. When comparing reconstructions obtained using the same number of projections, the porous regions, for example within the rectangle, are better defined in case of the EDART(calc) reconstruction.

\section{Conclusions}

In this paper a method for reconstructing experimental 3D datasets obtained with high resolution X-ray tomography, for samples consisting only of one material and surrounding (and enclosed) air was presented. This method is referred to as Experimental Discrete Algebraic Reconstruction Technique (EDART) and focuses on improving reconstruction quality in case few projections are present, without significantly increasing the reconstruction time. Additionally, a fast method to estimate the attenuation coefficient and threshold of the material in case these are unknown was presented. The results of the application of EDART on experimental samples were presented, these illustrate that EDART allows for the improvement of the reconstruction quality in case of limited projections in comparison with iterative reconstruction algorithms such as SART and that the 

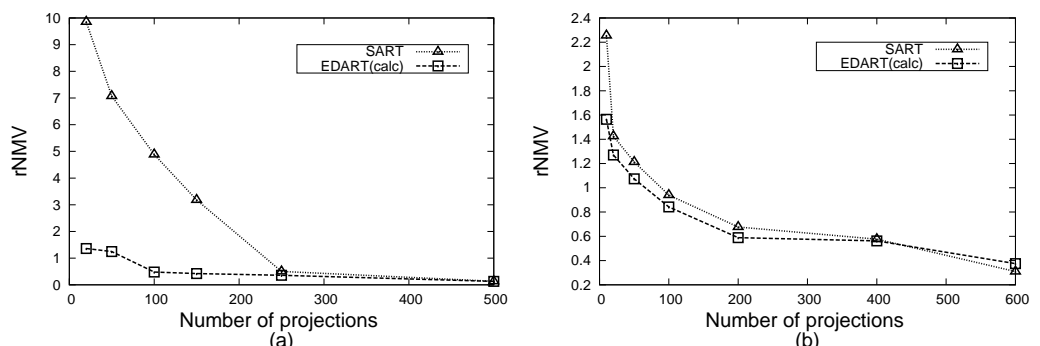

(a)

(b)

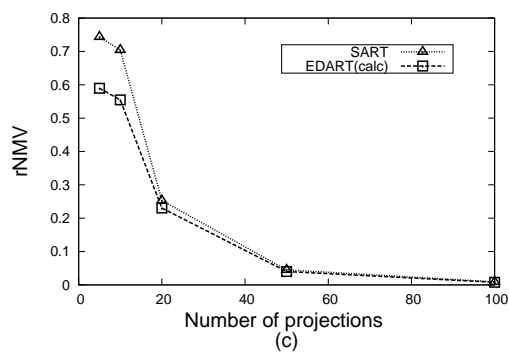

Figure 6: The $r N M V$ of the aluminum foam sample (a), the sample of cookies (b) and cavity in the toy sample (c) for SART and EDART(calc) reconstructions ( $\mu$ and $T$ as mentioned in Table 2 ) using various number of projections.

increase in reconstruction time is limited.

\section{Acknowledgements}

The Special Research Fund of the Ghent University (BOF) is acknowledged for the doctoral grant to Loes Brabant and the financial support (GOA 01G01008).

\section{References}

[1] G.T. Herman and A. Kuba. Discrete Tomography: Foundations, Algorithms, and Applications. Boston, MA: Birkhäuser, 1999.

[2] G.T. Herman and A. Kuba. Advances in Discrete Tomography and its Applications. Applied and Numerical Harmonic Analysis, 2005.

[3] T. Schüle, C. Schnörr, S. Weber, and J. Hornegger. Discrete tomography by convex-concave regularization and d.c. programming. Discrete Appl. Math., 151:229-243, 2005. 

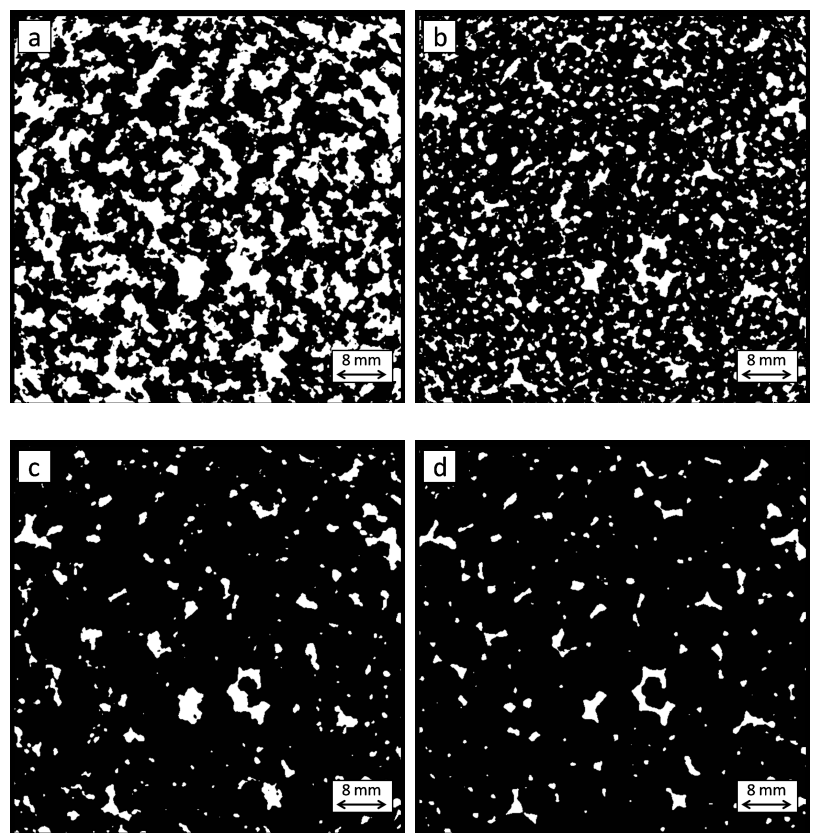

Figure 7: Same reconstructed cross section of the aluminum foam sample as that in Figure 4(b) in case of SART using $N_{p}=50$ (a), $N_{p}=150$ (b) and EDART(calc) using $N_{p}=50$ (c) and $N_{p}=150$ (d)

[4] H. Y. Liao and G. T. Herman. A coordinate ascent approach to tomographic reconstruction of label images from a few projections. Discrete Appl. Math., 151:184-197, 2005.

[5] K.J. Batenburg. A network flow algorithm for reconstructing binary images from continuous X-rays. J. Math. Imaging Vis., 30(3):231-248, 2008.

[6] X. Li and J. Zhu. A note of reconstruction algorithm for the strip-based projection model in discrete tomography. J. X-Ray Sci. Technol, 16:253260, 2008.

[7] J. Wang and L. Xing. A binary image reconstruction technique for accurate determination of the shape and location of metal objects in x-ray computed tomography. J. X-Ray Sci. Technol, 18(4):403-14, 2010.

[8] Q. Xu, E. Y. Sidky, X. Pan, M. Stampanoni, P. Modregger, and M. A. Anastasio. Investigation of discrete imaging models and iterative image reconstruction in differential X-ray phase-contrast tomography. Opt. Exp., 20(10):10724-10749, MAY 72012.

[9] K. Sen Sharma, X. Jin, C. Holzner, S. Narayanan, B. Liu, D. Wang, M. Agah, L. Wang, H. Yu, and G. Wang. Experimental studies on few- 

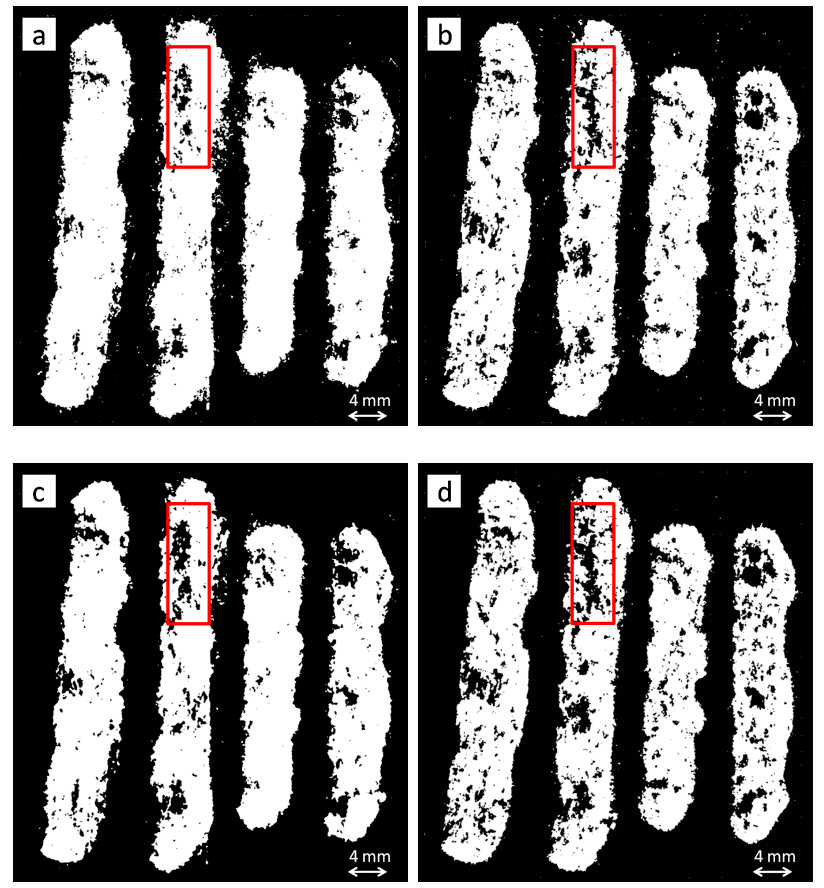

Figure 8: Same reconstructed cross section of the sample of cookies as that in Figure 5(b) in case of SART using $N_{p}=20$ (a), $N_{p}=50$ (b) and EDART(calc) using $N_{p}=20$ (c) and $N_{p}=50$ (d)

view reconstruction for high-resolution micro-ct. J. X-Ray Sci. Technol, 21:25-42, 2013.

[10] K.J. Batenburg and J. Sijbers. DART: A practical reconstruction algorithm for discrete tomography. IEEE Trans. Image Process., 20(9):2542-2553, 2011.

[11] K. J. Batenburg, S. Bals, J. Sijbers, C. Kuebel, P. A. Midgley, J. C. Hernandez, U. Kaiser, E. R. Encina, E. A. Coronado, and G. Van Tendeloo. 3d imaging of nanomaterials by discrete tomography. Ultramicroscopy, 109(6):730-740, MAY 2009.

[12] K. J. Batenburg, J. Sijbers, H. F. Poulsen, and E. Knudsen. DART: a robust algorithm for fast reconstruction of three-dimensional grain maps. J. Appl. CRrustallogr., 43(Part 6):1464-1473, DEC 2010.

[13] T. Roelandts, K. J. Batenburg, E. Biermans, C. Kuebel, S. Bals, and J. Sijbers. Accurate segmentation of dense nanoparticles by partially discrete electron tomography. Ultramicroscopy, 114:96-105, MAR 2012. 
[14] A.P. Dempster, N.M. Laird, and D.B. Rubin. Maximum likelihood from incomplete data via EM algorithm. J. Roy. Statist. Soc. B, 39(1):1-38, 1977.

[15] A.H. Andersen and A.C. Kak. Simultaneous algebraic reconstruction technique (SART) - a superior implementation of the ART algorithm. Ultranson. Imag., 6(1):81-94, 1984.

[16] R. Rangayyan, A.P. Dhawan, and R. Gordon. Algorithms for limited-view computed tomography - an annotated-bibliography and a challenge. Appl. Opt., 24(23):4000-4012, 1985.

[17] H.M. Hudson and R.S. Larkin. Accelerated image-reconstruction using ordered subsets of projection data. IEEE Trans. Med. Imag., 13(4):601609, DEC 1994.

[18] C.L. Byrne. Accelerating the EMML algorithm and related iterative algorithms by rescaled block-iterative methods. IEEE Trans. Image Process., 7(1):100-109, JAN 1998.

[19] F. Natterer. The mathematics of computerized tomography. Society for Industrial and Applied Mathematics, Philadelphia, PA, USA, 2001.

[20] J.S. Kole. Statistical image reconstruction for transmission tomography using relaxed ordered subset algorithms. Phys. Med. Biol., 50(7):15331545, APR 72005.

[21] L. Zeng, B. Liu, L. Liu, and C. Xiang. A new iterative reconstruction algorithm for 2d exterior fan-beam ct. J. X-Ray Sci. Technol, 18:267-277, 2010.

[22] M. Defrise, C. Vanhove, and X. Liu. An algorithm for total variation regularization in high-dimensional linear problems. Inverse Probl., 27(6), JUN 2011.

[23] L. Jian, L. Litao, C. Peng, S. Qi, and W. Zhifang. Rotating polarcoordinate art applied in industrial $\mathrm{CT}$ image reconstruction. NDT \& $\mathrm{E}$ Int., 40(4):333-336, JUN 2007.

[24] N. Jain, A. Raj, M. S. Kalra, P. Munshi, and V. R. Ravindran. MART algorithms for circular and helical cone-beam tomography. Res. Nondestruct. Eval., 22(3):147-168, 2011.

[25] X. Zhao, J.J. Hu, and T. Yang. Gpu based iterative cone-beam ct reconstruction using empty space skipping technique. J. X-Ray Sci. Technol, 21:53-69, 2013.

[26] L.A. Feldkamp, L.C. Davis, and J.W. Kress. Practical cone-beam algorithm. J. Opt. Soc. Amer. A, Opt. Image Sci., 1(6):612-619, 1984. 
[27] S. Valton, F. Peyrin, and D. Sappey-Marinier. Analysis of cone-beam artifacts in off-centered circular CT for four reconstruction methods. Int. J. Biomed. Imaging., 2006:1-8, 2006.

[28] Y. De Witte. Improved and Practically Feasible Reconstruction Methods for High Resolution X-Ray Microtomography. PhD thesis, Ghent University, 2010 .

[29] B. De Man, J. Nuyts, P. Dupont, G. Marchal, and P. Suetens. Reduction of metal streak artifacts in X-ray computed tomography using a transmission maximum a posteriori algorithm. IEEE Trans. Nucl. Sci., 47(3):977-981, 2000 .

[30] I. A. Elbakri and J. A. Fessler. Statistical image reconstruction for polyenergetic X-ray computed tomography. IEEE Trans. Med. Imag., 21(2):89-99, 2002 .

[31] J. Hsieh, R. C. Molthen, C. A. Dawson, and R. H. Johnson. An iterative approach to the beam hardening correction in cone beam CT. Med. Phys., 27(1):23-29, 2000.

[32] G. Van Gompel, K. Van Slambrouck, M. Defrise, K. J. Batenburg, J. de Mey, J. Sijbers, and J. Nuyts. Iterative correction of beam hardening artifacts in CT. Med. Phys., 38(1):S36-S49, JUL 2011.

[33] L. Brabant, E. Pauwels, M. Dierick, D. Van Loo, M.A. Boone, and L. Van Hoorebeke. A novel beam hardening correction method requiring no prior knowledge, incorporated in an iterative reconstruction algorithm. NDT $\mathcal{E}$ E Int., 51(0):68 - 73, 2012.

[34] A. H. Andersen. Algebraic reconstruction in CT from limited views. IEEE Trans. Med. Imag., 8(1):50-5, 1989.

[35] G. Wang, M.W. Vannier, and P.C. Cheng. Iterative X-ray cone-beam tomography for metal artifact reduction and local region reconstruction. Microsc. Microanal., 5(1):58-65, JAN-FEB 1999.

[36] E. Y. Sidky, C.-M. Kao, and X. Pan. Accurate image reconstruction from few-views and limited-angle data in divergent-beam CT. J. X-Ray Sci. Technol., 14(2):119-139, 2006.

[37] S. Peng and Z. Wu. Research on the CT image reconstruction of steel tube section from few projections. NDT $\&$ E Int., 42(5):435 - 440, 2009.

[38] K.J. Batenburg and J. Sijbers. Optimal threshold selection for tomogram segmentation by projection distance minimization. IEEE Trans. Med. Imag., 28(5):676-686, 2009.

[39] K.J. Batenburg, W. van Aarle, and J. Sijbers. A semi-automatic algorithm for grey level estimation in tomography. Pat. Rec. Let., 32(9):1395-1405, 2011. 
[40] W. van Aarle, K.J. Batenburg, and J. Sijbers. Automatic parameter estimation for the discrete algebraic reconstruction technique (DART). IEEE Trans. Image Process., 21(11):4608-4621, 2012.

[41] N. Otsu. A threshold selection method from gray-level histograms. IEEE Trans. Syst. Man Cybern., 9(1):62-66, January 1979.

[42] Deng-Yuan Huang and Chia-Hung Wang. Optimal multi-level thresholding using a two-stage otsu optimization approach. Pattern Recogn. Lett., 30(3):275-284, February 2009.

[43] B. C. Masschaele, V. Cnudde, M. Dierick, P. Jacobs, L. Van Hoorebeke, and J. Vlassenbroeck. UGCT: New X-ray radiography and tomography facility. Nucl. Instrum. Methods Phys. Res., Sect. A, 580(1):266-269, SEP 212007. 